\title{
Decreasing Diagrams and Relative Termination ${ }^{\star}$
}

\author{
Nao Hirokawa ${ }^{1}$ and Aart Middeldorp ${ }^{2}$ \\ 1 School of Information Science \\ Japan Advanced Institute of Science and Technology, Japan \\ 2 Institute of Computer Science \\ University of Innsbruck, Austria
}

\begin{abstract}
In this paper we use the decreasing diagrams technique to show that a left-linear term rewrite system $\mathcal{R}$ is confluent if all its critical pairs are joinable and the critical pair steps are relatively terminating with respect to $\mathcal{R}$. We further show how to encode the rule-labeling heuristic for decreasing diagrams as a satisfiability problem. Experimental data for both methods are presented.
\end{abstract}

\section{Introduction}

This paper is concerned with automatically proving confluence of term rewrite systems. Unlike termination, for which the interest in automation gave and continues to give rise to new methods and tools, ${ }^{3}$ automating confluence has received little attention. Only very recently, the first confluence tool made its appearance: ACP [2] implements Knuth and Bendix' condition - joinability of critical pairsfor terminating rewrite systems [14], several critical pair criteria for left-linear rewrite systems $[11,19,21]$, as well as divide and conquer techniques based on persistence [1], layer-preservation [16], and commutativity [17].

For abstract rewrite systems, the decreasing diagrams technique of van Oostrom [20] subsumes all sufficient conditions for confluence. To use this technique for term rewrite systems, a well-founded order on the rewrite steps has to be supplied such that rewrite peaks can be completed into so-called decreasing diagrams.

We present two results in this paper. We show how to encode the rule-labeling heuristic of van Oostrom [22] for linear rewrite systems as a satisfiability problem. In this heuristic rewrite steps are labeled by the applied rewrite rule. By limiting the number of steps that may be used to complete local diagrams, we obtain a finite search problem which is readily transformed into a satisfiability problem. Any satisfying assignment returned by a modern SAT or SMT solver is then translated back into a concrete rule-labeling.

\footnotetext{
* The research described in this paper is the starting point of FWF (Austrian Science Fund) project P22467 and the Grant-in-Aid for Young Scientists (B) 22700009 of the Japan Society for the Promotion of Science.

${ }^{3}$ http://termination-portal .org/wiki/Termination_Competition
} 
Our second and main result employs the decreasing diagrams technique to obtain a new confluence result for left-linear but not necessarily right-linear rewrite systems. It requires that the rewrite steps involved in the generation of critical pairs are relatively terminating with respect to the rewrite system. This result can be viewed as a generalization of the two standard approaches for proving confluence: orthogonality and joinability of critical pairs for terminating systems. In the non-trivial proof we use the self-labeling heuristic in which rewrite steps are labeled by their starting term.

Throughout the paper we assume familiarity with the basics of term rewriting $([18])$. In the next section we recall the decreasing diagrams technique and present a small variation which better serves our purposes. Section 3 is devoted to our main result. We prove that a locally confluent left-linear term rewrite system is confluent if there are no infinite rewrite sequences that involve infinitely many steps that were used in the generation of critical pairs. In Section 4 we explain how this result is implemented. Moreover, we show how the rule-labeling heuristic for decreasing diagrams can be transformed into a satisfiability problem. Section 5 presents experimental data. In Section 6 we conclude with suggestions for future research.

\section{Decreasing Diagrams}

We start this preliminary section by recalling the decreasing diagrams technique for abstract rewrite systems (ARSs) from [20]. We write $\left\langle A,\left\{\rightarrow_{\alpha}\right\}_{\alpha \in I}\right\rangle$ to denote the $\operatorname{ARS}\langle A, \rightarrow\rangle$ where $\rightarrow$ is the union of $\rightarrow_{\alpha}$ for all $\alpha \in I$. If $J \subseteq I$ then $\rightarrow_{J}$ denotes the union of $\rightarrow \alpha$ for all $\alpha \in J$.

Let $\mathcal{A}=\left\langle A,\left\{\rightarrow_{\alpha}\right\}_{\alpha \in I}\right\rangle$ be an ARS and let $>$ be a well-founded order on $I$. For every $\alpha \in I$ we write $\stackrel{\vee}{\longrightarrow}_{\alpha}$ for the union of $\rightarrow_{\beta}$ for all $\beta<\alpha$. If $\alpha, \beta \in I$ then $\stackrel{\vee}{\longrightarrow}_{\alpha \beta}$ denotes the union of $\stackrel{\vee}{\longrightarrow}_{\alpha}$ and $\stackrel{\vee}{\longrightarrow}_{\beta}$. Moreover, we write $\stackrel{\vee}{\longrightarrow}_{\alpha \beta}^{*}$ for $\left(\stackrel{\vee}{\longrightarrow}_{\alpha \beta}\right)^{*}$. We say that $\alpha$ and $\beta$ are locally decreasing with respect to $>$ and we write $\mathrm{LD}_{>}(\alpha, \beta)$ if

$$
\alpha \leftarrow \cdot \rightarrow_{\beta} \subseteq \stackrel{\vee}{\longrightarrow}_{\alpha}^{*} \cdot \rightarrow_{\beta}^{\bar{\beta}} \cdot \stackrel{\vee}{\longrightarrow}_{\alpha \beta}^{*} \cdot{ }_{\alpha \beta}^{*} \stackrel{\vee}{\longleftarrow} \cdot \overline{\bar{\alpha}}_{\alpha}^{\leftarrow} \cdot{ }_{\beta}^{*} \stackrel{\vee}{\longleftarrow}
$$

Graphically (dashed arrows are implicitly existentially quantified and doubleheaded arrows denote reflexive and transitive closure):

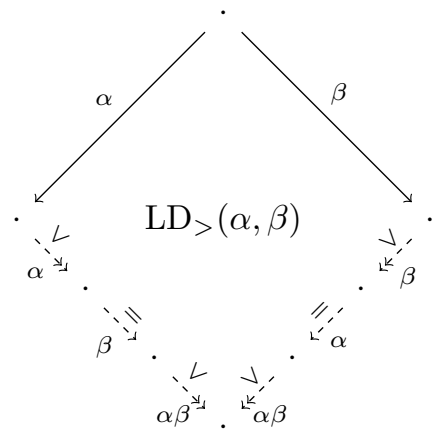


The ARS $\mathcal{A}=\left\langle A,\left\{\rightarrow_{\alpha}\right\}_{\alpha \in I}\right\rangle$ is locally decreasing if there exists a well-founded order $>$ on $I$ such that $\operatorname{LD}(\alpha, \beta)$ for all $\alpha, \beta \in I$.

Van Oostrom [20] obtained the following result.

Theorem 1. Every locally decreasing ARS is confluent.

Variations of this fundamental confluence result are presented in $[3,12,13,22]$. We present a version of Theorem 1 which is more suitable for our purposes.

Let $\mathcal{A}=\left\langle A,\left\{\rightarrow_{\alpha}\right\}_{\alpha \in I}\right\rangle$ be an ARS. Let $(>, \geqslant)$ consist of a well-founded order $>$ on $I$ together with a quasi-order $\geqslant$ such that $\geqslant \cdot>\cdot \geqslant \subseteq>$. For every $\alpha \in I$ we write $\stackrel{\mathbb{V}}{\longrightarrow}_{\alpha}$ for the union of $\rightarrow_{\beta}$ for all $\beta \leqslant \alpha$ and all $\beta<\alpha$. (Note that $>\subseteq \geqslant$ need not hold.) We say that $\alpha$ and $\beta$ are locally decreasing with respect to $(>, \geqslant)$ and we write $\operatorname{LD}_{(>, \geqslant)}(\alpha, \beta)$ if

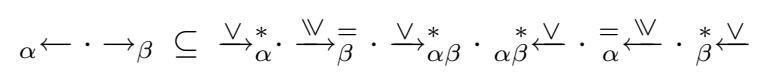

The ARS $\mathcal{A}=\left\langle A,\left\{\rightarrow_{\alpha}\right\}_{\alpha \in I}\right\rangle$ is extended locally decreasing if there exists (>, $\left.\geqslant\right)$ such that $\operatorname{LD}_{(>, \geqslant)}(\alpha, \beta)$ for all $\alpha, \beta \in I$. Despite the name, from the proof of the following theorem we infer that every extended locally decreasing ARS has a locally decreasing presentation.

Theorem 2. Every extended locally decreasing ARS is confluent.

Proof. Let $\mathcal{A}=\left\langle A,\left\{\rightarrow_{\alpha}\right\}_{\alpha \in I}\right\rangle$ be extended locally decreasing with respect to $(>, \geqslant)$. We write $C_{\alpha}$ as the set of all $\beta \in I$ with $\alpha \geqslant \beta$ and $\alpha \ngtr \beta$. The set of all such $C_{\alpha}$ is denoted by $\mathcal{C}$. For every $C \in \mathcal{C}$ we write $\rightarrow_{C}$ for the union of $\rightarrow_{\alpha}$ for all $\alpha \in C$. The well-founded order $>$ on $I$ can be lifted to $\mathcal{C}: C_{\alpha}>C_{\beta}$ if $\alpha>\beta$. If $C_{\alpha}=C_{\alpha^{\prime}}, C_{\beta}=C_{\beta^{\prime}}$, and $\alpha>\beta$ then $\alpha^{\prime} \geqslant \alpha>\beta \geqslant \beta^{\prime}$ and thus $\alpha^{\prime}>\beta^{\prime}$ because of the requirement $\geqslant \cdot>\cdot \geqslant \subseteq>$. Hence $>$ is well-defined on $\mathcal{C}$. If $\beta \leqslant \alpha$ and $\beta<\alpha$ then $\rightarrow_{\beta} \subseteq \rightarrow_{C_{\beta}} \subseteq \underline{v}_{C_{\alpha}}$. If $\beta \leqslant \alpha$ and $\beta \nless \alpha$ then $\beta \in C_{\alpha}$ and thus $\rightarrow_{\beta} \subseteq \rightarrow_{C_{\alpha}}$. Hence $\stackrel{\mathbb{V}^{\mathbb{V}}}{\longrightarrow} \subseteq \stackrel{\mathcal{V}}{\longrightarrow}_{C_{\alpha}} \cup \rightarrow_{C_{\alpha}}$. Now consider arbitrary sets $C, D \in \mathcal{C}$ and let $\alpha \in C$ and $\beta \in D$. From the assumption $\operatorname{LD}_{(>, \geqslant)}(\alpha, \beta)$ we obtain

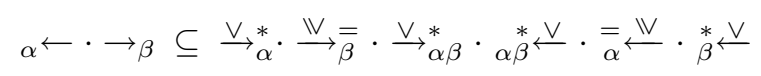

By construction, the latter relation is contained in

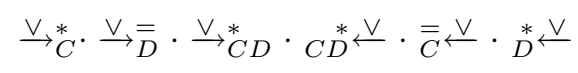

Since

$$
C \leftarrow \cdot \rightarrow_{D}=\bigcup_{\alpha \in C, \beta \in D} \alpha \leftarrow \cdot \rightarrow \beta
$$

we conclude $\mathrm{LD}_{>}(C, D)$. According to Theorem 1, the ARS $\left\langle A,\left\{\rightarrow_{C}\right\}_{C \in \mathcal{C}}\right\rangle$ is confluent. Since

$$
\bigcup_{\alpha \in I} \rightarrow_{\alpha}=\bigcup_{C \in \mathcal{C}} \rightarrow_{C}
$$

it follows that $\mathcal{A}$ is confluent. 
We are interested in the application of Theorems 1 and 2 for proving confluence of term rewrite systems (TRSs). Many sufficient conditions for confluence of TRSs are based on critical pairs. Critical pairs are generated from overlaps. An overlap $\left(l_{1} \rightarrow r_{1}, p, l_{2} \rightarrow r_{2}\right)_{\mu}$ of a TRS $\mathcal{R}$ consists of variants $l_{1} \rightarrow r_{1}$ and $l_{2} \rightarrow r_{2}$ of rules of $\mathcal{R}$ without common variables, a position $p \in \mathcal{P o s}_{\mathcal{F}}\left(l_{2}\right)$, and a most general unifier $\mu$ of $l_{1}$ and $\left.l_{2}\right|_{p}$. If $p=\epsilon$ then we require that $l_{1} \rightarrow r_{1}$ and $l_{2} \rightarrow r_{2}$ are not variants. The induced critical pair is $\left(l_{2} \mu\left[r_{1} \mu\right]_{p}, r_{2} \mu\right)$. Following Dershowitz [5], we write $s \leftarrow \rtimes \rightarrow t$ to indicate that $(s, t)$ is a critical pair.

In $[22]$ van Oostrom proposed the rule-labeling heuristic in which rewrite steps are partitioned according to the employed rewrite rules. If one can find an order on the rules of a linear TRS such that every critical pair is locally decreasing, confluence is guaranteed. A formalization of this heuristic is given below where $\alpha \leftarrow \rtimes \rightarrow \beta$ denotes the set of critical pairs obtained from overlaps $(\alpha, p, \beta)_{\mu}$.

Theorem 3. A linear TRS $\mathcal{R}$ is confluent if there exists a well-founded order

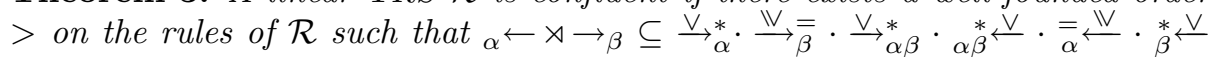
for all rewrite rules $\alpha, \beta \in \mathcal{R}$. Here $\geqslant$ is the reflexive closure of $>$.

The heuristic readily applies to the following example from [10].

Example 4. Consider the linear TRS $\mathcal{R}$ consisting of the rewrite rules

$$
\begin{aligned}
& \text { 1: } \quad \text { nats } \rightarrow 0: \operatorname{inc}(\text { nats) } \quad 4: \quad \operatorname{inc}(x: y) \rightarrow \mathrm{s}(x): \operatorname{inc}(y) \\
& 2: \operatorname{hd}(x: y) \rightarrow x \quad 5: \operatorname{inc}(\operatorname{tl}(\text { nats })) \rightarrow \operatorname{tl}(\text { inc(nats)) } \\
& \text { 3: } \operatorname{tl}(x: y) \rightarrow y
\end{aligned}
$$

There is one critical pair: inc $(\mathrm{tl}(0: \operatorname{inc}($ nats $))) \stackrel{1}{\leftarrow} \operatorname{inc}(\mathrm{tl}($ nats $)) \stackrel{5}{\rightarrow} \mathrm{tl}(\operatorname{inc}($ nats $))$. We have

$$
\begin{array}{r}
\operatorname{inc}(\mathrm{tl}(0: \operatorname{inc}(\text { nats }))) \stackrel{3}{\rightarrow} \operatorname{inc}(\operatorname{inc}(\text { nats })) \\
\mathrm{tl}(\operatorname{inc}(\text { nats })) \stackrel{1}{\rightarrow} \mathrm{tl}(\operatorname{inc}(0: \operatorname{inc}(\text { nats }))) \stackrel{4}{\rightarrow} \mathrm{tl}(\mathrm{s}(0): \operatorname{inc}(\operatorname{inc}(\text { nats }))) \stackrel{3}{\rightarrow} \operatorname{inc}(\text { inc }(\text { nats }))
\end{array}
$$

Hence the critical pair is locally decreasing with respect to the rule-labeling heuristic together with the order $5>3,4$.

The following example (Vincent van Oostrom, personal communication) shows that linearity in Theorem 3 cannot be weakened to left-linearity.

Example 5. Consider the TRS $\mathcal{R}$ consisting of the rewrite rules

$$
1: \mathrm{f}(\mathrm{a}, \mathrm{a}) \rightarrow \mathrm{c} \quad 2: \mathrm{f}(\mathrm{b}, x) \rightarrow \mathrm{f}(x, x) \quad 3: \mathrm{f}(x, \mathrm{~b}) \rightarrow \mathrm{f}(x, x) \quad 4: \mathrm{a} \rightarrow \mathrm{b}
$$

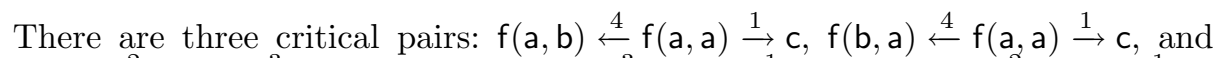
$\mathrm{f}(\mathrm{b}, \mathrm{b}) \stackrel{2}{\leftarrow} \mathrm{f}(\mathrm{b}, \mathrm{b}) \stackrel{3}{\rightarrow} \mathrm{f}(\mathrm{b}, \mathrm{b})$. Since $\mathrm{f}(\mathrm{a}, \mathrm{b}) \stackrel{3}{\rightarrow} \mathrm{f}(\mathrm{a}, \mathrm{a}) \stackrel{1}{\rightarrow} \mathrm{c}$ and $\mathrm{f}(\mathrm{b}, \mathrm{a}) \stackrel{2}{\rightarrow} \mathrm{f}(\mathrm{a}, \mathrm{a}) \stackrel{1}{\rightarrow} \mathrm{c}$, it follows that the critical pairs are locally decreasing by taking the order $4>2,3$. Nevertheless, the conversion $\mathrm{f}(\mathrm{b}, \mathrm{b}) \leftarrow \mathrm{f}(\mathrm{b}, \mathrm{a}) \leftarrow \mathrm{f}(\mathrm{a}, \mathrm{a}) \rightarrow \mathrm{c}$ reveals that $\mathcal{R}$ is not confluent.

In the next section we impose a relative termination condition to obtain a confluence criterion for possibly non-right-linear TRSs. 


\section{Confluence via Relative Termination}

Let $\mathcal{R}$ be a TRS. We denote the set

$$
\left\{l_{2} \mu \rightarrow l_{2} \mu\left[r_{1} \mu\right]_{p}, l_{2} \mu \rightarrow r_{2} \mu \mid\left(l_{1} \rightarrow r_{1}, p, l_{2} \rightarrow r_{2}\right)_{\mu} \text { is an overlap of } \mathcal{R}\right\}
$$

of rewrite steps that give rise to critical pairs of $\mathcal{R}$ by $\operatorname{CPS}(\mathcal{R})$. The rules in $\operatorname{CPS}(\mathcal{R})$ are called critical pair steps. We say that $\mathcal{R}$ is relatively terminating with respect to $\mathcal{S}$ or that $\mathcal{R} / \mathcal{S}$ is terminating if the relation $\rightarrow_{\mathcal{R} / \mathcal{S}}=\rightarrow_{\mathcal{S}}^{*} \cdot \rightarrow_{\mathcal{R}} \cdot \rightarrow_{\mathcal{S}}^{*}$ is well-founded. The main result of this section (Theorem 16 below) states that a left-linear locally confluent TRS $\mathcal{R}$ is confluent if $\operatorname{CPS}(\mathcal{R})$ is relatively terminating with respect to $\mathcal{R}$. Since $\operatorname{CPS}(\mathcal{R})$ is empty for every orthogonal TRS $\mathcal{R}$, this yields a generalization of orthogonality. In the proof we use decreasing diagrams with the self-labeling heuristic in which rewrite steps are labeled by their starting term. A key problem when trying to prove confluence in the absence of termination is the handling of duplicating rules. Parallel rewrite steps are typically used for this purpose $[11,17]$. To anticipate future developments (cf. Section 6) we use multi-steps instead. However, first we present a special case of our main result in which duplicating rules are taken care of by requiring them to be relatively terminating with respect to the non-duplicating ones.

Theorem 6. Let $\mathcal{R}$ be a left-linear TRS. Let $\mathcal{R}_{\mathrm{d}}$ be the subset of duplicating rules and $\mathcal{R}_{\text {nd }}$ the subset of non-duplicating rules in $\mathcal{R}$. The TRS $\mathcal{R}$ is confluent if $\leftarrow \rtimes \rightarrow \subseteq \downarrow$ and $\operatorname{CPS}(\mathcal{R}) \cup \mathcal{R}_{\mathrm{d}}$ is relatively terminating with respect to $\mathcal{R}_{\mathrm{nd}}$.

Proof. We label rewrite steps by their starting term. Labels are compared with respect to the strict order $>=\rightarrow_{\left(\mathrm{CPS}(\mathcal{R}) \cup \mathcal{R}_{\mathrm{d}}\right) / \mathcal{R}_{\text {nd }}}^{+}$and the quasi-order $\geqslant=\rightarrow_{\mathcal{R}}^{*}$. Note that $>$ is well-founded by the assumption that $\operatorname{CPS}(\mathcal{R}) \cup \mathcal{R}_{\mathrm{d}}$ is relatively terminating with respect to $\mathcal{R}_{\text {nd }}$. We show that all local peaks of $\mathcal{R}$ are extended locally decreasing. Let $s \rightarrow t_{1}$ and $s \rightarrow t_{2}$ by applying the rewrite rules $l_{1} \rightarrow r_{1}$ and $l_{2} \rightarrow r_{2}$ at the positions $p_{1}$ and $p_{2}$. We may assume that $l_{1} \rightarrow r_{1}$ and $l_{2} \rightarrow r_{2}$ do not share variables and thus there exists a substitution $\sigma$ such that $s=s\left[l_{1} \sigma\right]_{p_{1}}=s\left[l_{2} \sigma\right]_{p_{2}}, t_{1}=s\left[r_{1} \sigma\right]_{p_{1}}$, and $t_{2}=s\left[r_{2} \sigma\right]_{p_{2}}$. We distinguish three cases.

1. If $p_{1} \| p_{2}$ then $t_{1} \rightarrow u \leftarrow t_{2}$ for the term $u=s\left[r_{1} \sigma, r_{2} \sigma\right]_{p_{1}, p_{2}}$. We have $s>t_{1}$ if $l_{1} \rightarrow r_{1}$ is duplicating and $s \geqslant t_{1}$ if $l_{1} \rightarrow r_{1}$ is non-duplicating. So in both cases we have $t_{1} \stackrel{\mathbb{V}}{\longrightarrow} u$. Similarly, $t_{2} \stackrel{\mathbb{V}}{\longrightarrow} s u$ and thus we have local decreasingness.

2. Suppose the redexes $l_{1} \sigma$ at position $p_{1}$ and $l_{2} \sigma$ at position $p_{2}$ overlap. If $p_{1}=$ $p_{2}$ and $l_{1} \rightarrow r_{1}$ and $l_{2} \rightarrow r_{2}$ are variants then $t_{1}=t_{2}$ and there is nothing to prove. Assume without loss of generality that $p_{1} \leqslant p_{2}$. There exists a substitution $\tau$ such that $t_{1}=s[v \tau]_{p_{1}}$ and $t_{2}=s[u \tau]_{p_{1}}$ with $u \leftarrow \rtimes \rightarrow v$. By assumption $u \downarrow v$ and hence also $t_{1} \downarrow t_{2}$. Every label $a$ in the valley between $t_{1}$ and $t_{2}$ satisfies $t_{1} \geqslant a$ or $t_{2} \geqslant a$. Since $s \rightarrow \operatorname{CPS}(\mathcal{R}) t_{1}$ and $s \rightarrow \operatorname{CPS}(\mathcal{R}) t_{2}$, it follows that $s>t_{1}, t_{2}$. Hence $s>a$ for every label $a$ in the valley between $t_{1}$ and $t_{2}$. Consequently, local decreasingness holds. 
3. In the remaining case we have a variable overlap. Assume without loss of generality that $p_{1}<p_{2}$. Let $x$ be the variable in $l_{1}$ whose position is above $p_{2} \backslash p_{1}$. Due to linearity of $l_{1}$ we have $t_{1} \rightarrow^{*} u \leftarrow t_{2}$ for some term $u$. The number of steps in the sequence from $t_{1}$ to $u$ equals the number of occurrences of the variable $x$ in $r_{1}$. If this number is not more than one then local decreasingness is obtained as in the first case. If this number is more than one then $l_{1} \rightarrow r_{1}$ is duplicating and hence $s>t_{1}$. Therefore $s>a$ for every term $a$ in the sequence from $t_{1}$ to $u$. Moreover $s>t_{2}$ or $s \geqslant t_{2}$. Hence also in this case we have local decreasingness.

Example \%. Consider the TRS $\mathcal{R}$ from [9, p.28] consisting of the rewrite rules

$$
\mathrm{f}(\mathrm{g}(x)) \rightarrow \mathrm{f}(\mathrm{h}(x, x)) \quad \mathrm{g}(\mathrm{a}) \rightarrow \mathrm{g}(\mathrm{g}(\mathrm{a})) \quad \mathrm{h}(\mathrm{a}, \mathrm{a}) \rightarrow \mathrm{g}(\mathrm{g}(\mathrm{a}))
$$

The only critical pair $\mathrm{f}(\mathrm{g}(\mathrm{g}(\mathrm{a}))) \leftarrow \rtimes \rightarrow \mathrm{f}(\mathrm{h}(\mathrm{a}, \mathrm{a}))$ is clearly joinable. The TRS $\operatorname{CPS}(\mathcal{R}) \cup \mathcal{R}_{\mathrm{d}}$ consists of the rewrite rules

$$
\mathrm{f}(\mathrm{g}(\mathrm{a})) \rightarrow \mathrm{f}(\mathrm{h}(\mathrm{a}, \mathrm{a})) \quad \mathrm{f}(\mathrm{g}(\mathrm{a})) \rightarrow \mathrm{f}(\mathrm{g}(\mathrm{g}(\mathrm{a}))) \quad \mathrm{f}(\mathrm{g}(x)) \rightarrow \mathrm{f}(\mathrm{h}(x, x))
$$

and can be shown to be relatively terminating with respect to $\mathcal{R}_{\text {nd }}$ using the method described at the beginning of Section 4. Hence the confluence of $\mathcal{R}$ is concluded by Theorem 6 .

The following example ${ }^{4}$ shows that left-linearity is essential in Theorem 6 .

Example 8. Consider the non-left-linear TRS $\mathcal{R}$

$$
\mathrm{f}(x, x) \rightarrow \mathrm{a} \quad \mathrm{f}(x, \mathrm{~g}(x)) \rightarrow \mathrm{b} \quad \mathrm{c} \rightarrow \mathrm{g}(\mathrm{c})
$$

from [11]. Since $\operatorname{CPS}(\mathcal{R}) \cup \mathcal{R}_{\mathrm{d}}$ is empty, termination of $\left(\operatorname{CPS}(\mathcal{R}) \cup \mathcal{R}_{\mathrm{d}}\right) / \mathcal{R}_{\text {nd }}$ is trivial. However, $\mathcal{R}$ is not confluent because the term $f(c, c)$ has two distinct normal forms.

The termination of $\left(\mathrm{CPS}(\mathcal{R}) \cup \mathcal{R}_{\mathrm{d}}\right) / \mathcal{R}_{\text {nd }}$ can be weakened to the termination of $\operatorname{CPS}(\mathcal{R}) / \mathcal{R}$. In the proof of Theorem 6 we showed the local decreasingness of $\rightarrow \mathcal{R}$. The following example shows that this no longer holds under the weakened termination assumption.

Example 9. Consider the orthogonal TRS $\mathcal{R}$ consisting of the two rules $\mathrm{f}(x) \rightarrow$ $\mathrm{g}(x, x)$ and $\mathrm{a} \rightarrow \mathrm{b}$. Consider the local peak $\mathrm{f}(\mathrm{b}) \leftarrow \mathrm{f}(\mathrm{a}) \rightarrow \mathrm{g}(\mathrm{a}, \mathrm{a})$. There are two ways to complete the diagram:
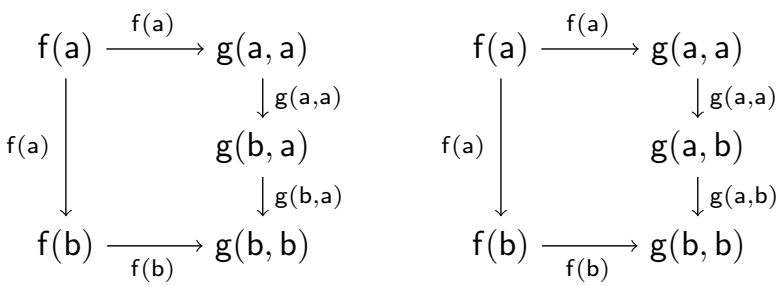

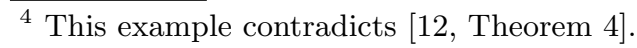


Since CPS $(\mathcal{R})$ is empty, neither of them is extended locally decreasing with respect to the order in the proof of Theorem 6 .

To address the problem, we first recall multi-steps (cf. [18]).

Definition 10. Let $\mathcal{R}$ be a TRS. The multi-step relation $\rightarrow \mathcal{R}$ (or simply $\rightarrow$ ) is inductively defined as follows:

(1) $x \rightarrow_{\mathcal{R}} x$ for all variables $x$,

(2) $f\left(s_{1}, \ldots, s_{n}\right) \rightarrow \rightarrow_{\mathcal{R}} f\left(t_{1}, \ldots, t_{n}\right)$ if for each $i$ we have $s_{i} \rightarrow \rightarrow_{\mathcal{R}} t_{i}$, and

(3) $l \sigma \rightarrow \rightarrow_{\mathcal{R}} r \tau$ if $l \rightarrow r \in \mathcal{R}$ and $\sigma \rightarrow_{\mathcal{R}} \tau$.

where $\sigma \rightarrow_{\mathcal{R}} \tau$ if $x \sigma \rightarrow_{\mathcal{R}} x \tau$ for all variables $x$.

The following result is well-known ([18, Lemma 4.7.12]).

Lemma 11. For every $T R S \mathcal{R}$ we have $\rightarrow_{\mathcal{R}} \subseteq \bullet_{\mathcal{R}} \subseteq \rightarrow_{\mathcal{R}}^{*}$.

The following lemma relates $\rightarrow \rightarrow_{\mathcal{R}}$ to $\rightarrow \operatorname{CPS}(\mathcal{R}) / \mathcal{R}$. It is the key to prove our main result.

Lemma 12. Let $\mathcal{R}$ be a TRS and $l \rightarrow r$ a left-linear rule in $\mathcal{R}$. If $l \sigma \rightarrow \rightarrow_{\mathcal{R}} t$ then one of the following conditions holds:

(a) $t \in\{l \tau, r \tau\}$ and $\sigma \rightarrow \mathcal{R} \tau$ for some $\tau$,

(b) $l \sigma \rightarrow \operatorname{CPS}(\mathcal{R}) \cdot \rightarrow \rightarrow_{\mathcal{R}} t$ and $l \sigma \rightarrow \operatorname{CPS}(\mathcal{R}) r \sigma$.

Proof. We may write $l \sigma=C\left[s_{1}, \ldots, s_{n}\right] \rightarrow_{\mathcal{R}} C\left[t_{1}, \ldots, t_{n}\right]=t$ where $s_{i} \rightarrow \rightarrow_{\mathcal{R}} t_{i}$ is obtained by case (3) in the definition of $\rightarrow_{\mathcal{R}}$ for all $1 \leqslant i \leqslant n$. If $n=0$ then $l \sigma=t$ and hence we can take $\tau=\sigma$ to satisfy condition (a). So let $n>0$. Let $p_{i}$ be the position of $s_{i}$ in $l \sigma$. We distinguish two cases.

- Suppose that $p_{1}, \ldots, p_{n} \notin \mathcal{P}_{\mathcal{F}}(l)$. We define a substitution $\tau$ as follows. For $x \in \operatorname{Var}(l)$ let $q$ be the (unique) position in $\operatorname{Pos}_{\mathcal{V}}(l)$ such that $\left.l\right|_{q}=x$. Let $P=\left\{p_{i} \mid p_{i} \geqslant q\right\}$ be the set of positions in $l \sigma$ of those terms $s_{1}, \ldots, s_{n}$ that occur in $\sigma(x)$. We define $\tau(x)$ as the term that is obtained from $\sigma(x)$ by replacing for all $p_{i} \in P$ the subterm $s_{i}$ at position $p_{i} \backslash q$ with $t_{i}$. We have $t=l \tau$ and $\sigma \rightarrow_{\mathcal{R}} \tau$, so condition (a) is satisfied.

- In the remaining case at least one position among $p_{1}, \ldots, p_{n}$ belongs to $\mathcal{P}_{\mathcal{F}}(l)$. Without loss of generality we assume that $p_{1} \in \mathcal{P}_{\operatorname{os}_{\mathcal{F}}}(l)$. Since $s_{1} \rightarrow \rightarrow_{\mathcal{R}} t_{1}$ is obtained by case (3), $s_{1}=l_{1} \mu$ and $t_{1}=r_{1} \nu$ for some rewrite rule $l_{1} \rightarrow r_{1}$ and substitutions $\mu$ and $\nu$ with $\mu \rightarrow_{\mathcal{R}} \nu$. We assume that $l_{1} \rightarrow r_{1}$ and $l \rightarrow r$ share no variables. Hence we may assume that $\mu=\sigma$. We distinguish two further cases.

- If $l_{1} \rightarrow r_{1}$ and $l \rightarrow r$ are variants and $p_{1}=\epsilon$ then $n=1, C=\square$, and $l \sigma=s_{1}=l_{1} \sigma \rightarrow_{\mathcal{R}} r_{1} \nu=t$. Because $l_{1} \rightarrow r_{1}$ and $l \rightarrow r$ are variants, there exists a substitution $\tau$ such that $r \tau=r_{1} \nu$ and $\sigma \rightarrow \mathcal{R} \tau$. So in this case condition (a) is satisfied. 
- If $l_{1} \rightarrow r_{1}$ and $l \rightarrow r$ are not variants or $p_{1} \neq \epsilon$ then there exists an overlap $\left(l_{1} \rightarrow r_{1}, p_{1}, l \rightarrow r\right)_{\theta}$ such that $l \sigma=l \sigma\left[l_{1} \sigma\right]_{p_{1}}$ is an instance of $l \theta=l \theta\left[l_{1} \theta\right]_{p_{1}}$. The TRS CPS $(\mathcal{R})$ contains the rules $l \theta \rightarrow l \theta\left[r_{1} \theta\right]_{p_{1}}$ and $l \theta \rightarrow r \theta$. The latter rule is used to obtain $l \sigma \rightarrow \operatorname{CPS}(\mathcal{R}) r \sigma$. An application of the former rule yields $l \sigma \rightarrow \operatorname{CPS}(\mathcal{R}) l \sigma\left[r_{1} \sigma\right]_{p_{1}}$. From $\sigma \rightarrow_{\mathcal{R}} \nu$ we infer that $r_{1} \sigma \rightarrow \rightarrow_{\mathcal{R}} r_{1} \nu=t_{1}$. Hence $l \sigma \rightarrow \operatorname{CPS}(\mathcal{R}) l \sigma\left[r_{1} \sigma\right]_{p_{1}} \rightarrow_{\mathcal{R}} l \sigma\left[t_{1}\right]_{p_{1}}=$ $C\left[t_{1}, s_{2}, \ldots, s_{n}\right] \rightarrow \rightarrow_{\mathcal{R}}^{*} C\left[t_{1}, \ldots, t_{n}\right]=t$. The $\multimap \rightarrow_{\mathcal{R}}$-steps can be combined into a single one and hence condition (b) is satisfied.

The following example shows that both conditions in Lemma 12 can occur.

Example 13. Consider the TRS $\mathcal{R}$ consisting of the rules $\mathrm{f}(\mathrm{g}(x), y) \rightarrow \mathrm{h}(x, y)$, $\mathrm{g}(\mathrm{a}) \rightarrow \mathrm{b}$, and $\mathrm{a} \rightarrow \mathrm{c}$. Let $l \rightarrow r$ be the first rule, $t=\mathrm{f}(\mathrm{b}, \mathrm{c})$, and $\sigma=\{x, y \mapsto \mathrm{a}\}$. We have $l \sigma \rightarrow \rightarrow_{\mathcal{R}} t$ with $t$ satisfying condition (b) in Lemma 12: $l \sigma \rightarrow \operatorname{CPS}(\mathcal{R})$ $\mathrm{f}(\mathrm{b}, \mathrm{a}) \rightarrow_{\mathcal{R}} t$ and $l \sigma \rightarrow \operatorname{CPS}(\mathcal{R}) r \sigma$. Note that condition (a) is not satisfied. If we take $t=\mathrm{f}(\mathrm{g}(\mathrm{a}), \mathrm{c})$ or $t=\mathrm{h}(\mathrm{g}(\mathrm{c}), \mathrm{c})$ then condition (a) is satisfied but condition (b) is not.

The following example shows the necessity of left-linearity in the preceding lemma.

Example 14. Consider the TRS $\mathcal{R}$ consisting of the rewrite rules $\mathrm{f}(x, x) \rightarrow \mathrm{b}$ and $\mathrm{a} \rightarrow \mathrm{b}$. Let $l \rightarrow r$ be the former rule, $t=\mathrm{f}(\mathrm{a}, \mathrm{b})$, and $\sigma=\{x \mapsto \mathrm{a}\}$. We have $l \sigma \rightarrow \rightarrow_{\mathcal{R}} t$ but $t$ satisfies neither condition in Lemma 12.

The final preliminary lemma states some obvious closure properties.

Lemma 15. Let $>$ and $\geqslant$ be closed under contexts.

1. If $t \stackrel{\vee}{\bullet} \underset{s}{\bullet} u$ then $C[t] \stackrel{\vee}{\bullet}{ }_{C[s]}^{*} C[u]$.

2. If $t \stackrel{\mathbb{\vee}}{\rightarrow}=$

3. Let $\geqslant=\rightarrow_{\mathcal{R}}^{*}$ and $\geqslant \cdot>\cdot \geqslant \subseteq$. If $s>t$ and $t \hookrightarrow^{*} u$ then $t \stackrel{\vee}{\leftrightarrow}{ }_{s}^{*} u$.

Proof. Straightforward.

After these preliminaries we are ready for the main result. In order to anticipate future developments (see Section 6), we avoid the use of advanced results from the confluence literature in the proof.

Theorem 16. A left-linear TRS $\mathcal{R}$ is confluent if $\leftarrow \rtimes \rightarrow \subseteq \downarrow$ and $\operatorname{CPS}(\mathcal{R}) / \mathcal{R}$ is terminating.

Proof. Because of Lemma 11, it is sufficient to prove confluence of $\rightarrow \rightarrow \mathcal{R}$. We show that the relation $\rightarrow \rightarrow \mathcal{R}$ is extended locally decreasing with respect to the source labeling. Labels are compared with respect to the strict order $>=$ $\rightarrow_{\mathrm{CPS}(\mathcal{R}) / \mathcal{R}}^{+}$and the quasi-order $\geqslant=\rightarrow_{\mathcal{R}}^{*}$. We show that

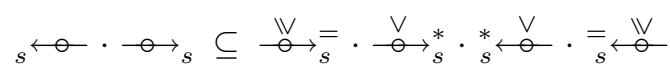

for all terms $s$ by well-founded induction on the order $(>\cup \triangleright)^{+}$. In the base case $s$ is a variable and the inclusion trivially holds. Let $s=f\left(s_{1}, \ldots, s_{n}\right)$. Suppose $t \leftarrow s \multimap u$. We distinguish the following cases, depending on the derivation of $s \rightarrow t$ and $s \rightarrow u$. 
- Neither $s \multimap t$ nor $s \multimap u$ is obtained by (1), because $s$ is not a variable. Suppose both $s \multimap t$ and $s \multimap u$ are obtained by (2). Then $t$ and $u$ can be written as $f\left(t_{1}, \ldots, t_{n}\right)$ and $f\left(u_{1}, \ldots, u_{n}\right)$. Fix $i \in\{1, \ldots, n\}$. We have $t_{i} \hookleftarrow s_{i} \multimap u_{i}$. By the induction hypothesis there exist $t_{i}^{\prime}, u_{i}^{\prime}$, and $v_{i}$ such that

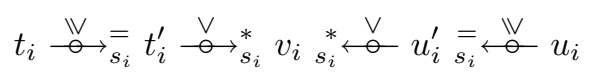

With repeated applications of Lemma 15(1,2) we obtain

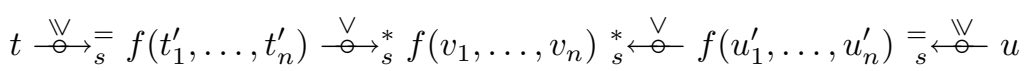

- Suppose $s \multimap t$ or $s \longrightarrow u$ is obtained by (3). Without loss of generality we assume that $s \rightarrow t$ is obtained by (3), i.e., $s=l \sigma, t=r \tau$, and $\sigma \multimap \tau$. Following Lemma 12, we distinguish the following two cases for $l \sigma \rightarrow u$.

- Suppose $u \in\{l \mu, r \mu\}$ for some $\mu$ with $\sigma \multimap \mu$. Fix $x \in \mathcal{V}$ ar $(l)$. We have $x \tau \hookleftarrow x \sigma \multimap x \mu$. By the induction hypothesis there exist terms $t_{x}$, $u_{x}$, and $v_{x}$ such that

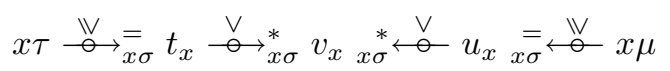

Define substitutions $\tau^{\prime}, \nu$, and $\mu^{\prime}$ as follows: $\tau^{\prime}(x)=t_{x}, \nu(x)=v_{x}$, and $\mu^{\prime}(x)=u_{x}$ for all $x \in \mathcal{V} \operatorname{ar}(l)$, and $\tau^{\prime}(x)=\nu(x)=\mu^{\prime}(x)=x$ for all $x \notin \mathcal{V}$ ar $(l)$. We obtain

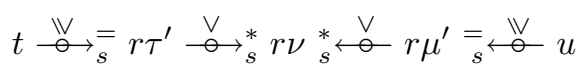

by repeated applications of Lemma $15(1,2)$.

- In the remaining case we have $s \rightarrow \operatorname{CPS}(\mathcal{R}) u^{\prime} \multimap u$ for some term $u^{\prime}$ as well as $s \rightarrow \operatorname{CPS}(\mathcal{R}) r \sigma$. Clearly $r \sigma \rightarrow r \tau=t$. Since $\mathcal{R}$ is locally confluent (due to $\leftarrow \rtimes \rightarrow \subseteq \downarrow$ ), there exists a term $v^{\prime}$ such that $r \sigma \rightarrow^{*} v^{\prime} \leftarrow u^{\prime}$ and thus also $r \sigma \bigcirc^{*} v^{\prime *} \leftarrow-u^{\prime}$. We have $s>r \sigma$ and $s>u^{\prime}$. Lemma 15(3) ensures that $r \sigma \stackrel{\vee}{\longrightarrow}{ }_{s}^{*} v^{\prime} \stackrel{*}{\stackrel{v}{\longleftarrow}} u^{\prime}$. For every term $v$ with $s>v$ we have

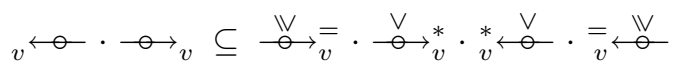

by the induction hypothesis. Hence the $\operatorname{ARS}\left\langle\mathcal{T}(\mathcal{F}, \mathcal{V}),\left\{\bullet_{v}\right\}_{v<s}\right\rangle$ is locally decreasing and therefore the relation

$$
\stackrel{\vee}{\rightarrow} s=\bigcup_{v<s} \multimap v
$$


is confluent. This is used to obtain the diagram

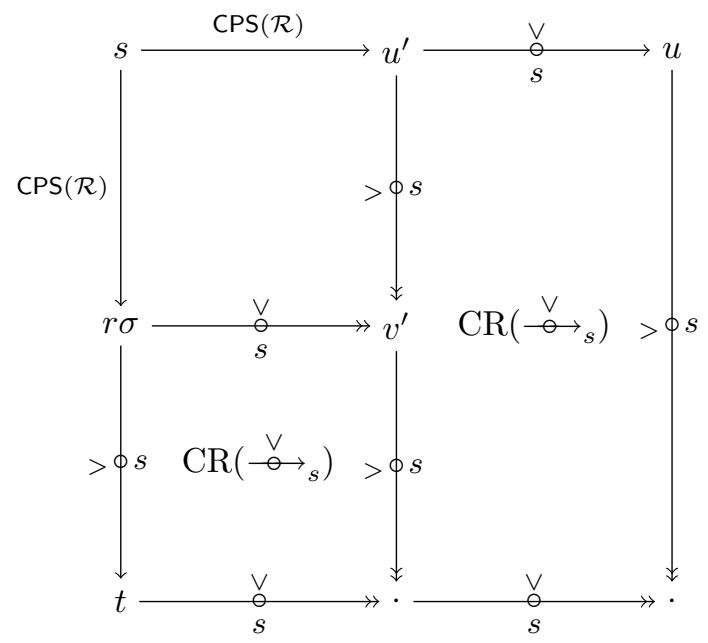

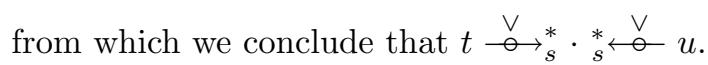

The above result can also be proved using Lemma 12 in connection with the conversion version of decreasing diagrams together with the predecessor labeling diagrams [22] in which steps $s \rightarrow t$ are labeled by terms $u$ such that $u \rightarrow^{*} s$ (Vincent van Oostrom, personal communication).

Example 17. Suppose we extend the TRS $\mathcal{R}$ of Example 4 by the rewrite rule

$$
\mathrm{d}(x: y) \rightarrow x:(x: \mathrm{d}(y))
$$

The resulting TRS $\mathcal{R}^{\prime}$ has the same critical pair as $\mathcal{R}$ and $\operatorname{CPS}\left(\mathcal{R}^{\prime}\right)$ consists of

$$
\operatorname{inc}(\operatorname{tl}(\text { nats })) \rightarrow \operatorname{tl}(\operatorname{inc}(\text { nats })) \quad \operatorname{inc}(\operatorname{tl}(\text { nats })) \rightarrow \operatorname{inc}(\operatorname{tl}(0: \operatorname{inc}(\text { nats })))
$$

By taking the matrix interpretation $([7])$

$$
\begin{aligned}
\operatorname{inc}_{\mathcal{M}}(\boldsymbol{x}) & =\left(\begin{array}{ll}
1 & 0 \\
1 & 0
\end{array}\right) \boldsymbol{x} & \operatorname{hd}_{\mathcal{M}}(\boldsymbol{x})=\boldsymbol{x} & 0_{\mathcal{M}}=\left(\begin{array}{l}
0 \\
0
\end{array}\right) \\
\operatorname{nats}_{\mathcal{M}} & =\left(\begin{array}{l}
0 \\
1
\end{array}\right) & \operatorname{tl}_{\mathcal{M}}(\boldsymbol{x})=\left(\begin{array}{ll}
1 & 1 \\
1 & 0
\end{array}\right) \boldsymbol{x} & \mathrm{s}_{\mathcal{M}}(\boldsymbol{x})=\left(\begin{array}{ll}
1 & 1 \\
0 & 0
\end{array}\right) \boldsymbol{x} \\
\mathrm{d}_{\mathcal{M}}(\boldsymbol{x}) & =\left(\begin{array}{ll}
1 & 1 \\
1 & 1
\end{array}\right) \boldsymbol{x} & :_{\mathcal{M}}(\boldsymbol{x}, \boldsymbol{y})=\left(\begin{array}{ll}
1 & 1 \\
1 & 1
\end{array}\right) \boldsymbol{x}+\boldsymbol{y} &
\end{aligned}
$$

we obtain $\mathcal{R}^{\prime} \subseteq \geqslant_{\mathcal{M}}$ and $\operatorname{CPS}\left(\mathcal{R}^{\prime}\right) \subseteq>_{\mathcal{M}}$ :

$$
[\operatorname{inc}(\operatorname{tl}(\text { nats }))]_{\mathcal{M}}=\left(\begin{array}{l}
1 \\
1
\end{array}\right)>\left(\begin{array}{l}
0 \\
0
\end{array}\right)=[\operatorname{tl}(\operatorname{inc}(\text { nats }))]_{\mathcal{M}}=[\operatorname{inc}(\operatorname{tl}(0: \operatorname{inc}(\text { nats })))]_{\mathcal{M}}
$$


Hence $\operatorname{CPS}\left(\mathcal{R}^{\prime}\right) / \mathcal{R}^{\prime}$ is terminating and Theorem 16 yields the confluence of $\mathcal{R}^{\prime}$. Note that Theorem 6 does not apply because $\operatorname{CPS}\left(\mathcal{R}^{\prime}\right) \cup \mathcal{R}_{\mathrm{d}}^{\prime}$ is not relatively terminating with respect to $\mathcal{R}_{\text {nd }}^{\prime}$; consider the term $\mathrm{d}$ (nats).

Replacing $\mathrm{CPS}(\mathcal{R})$ in Theorem 16 by $\mathrm{CPS}^{\prime}(\mathcal{R})=\left\{l_{1} \mu \rightarrow r_{1} \mu, l_{2} \mu \rightarrow r_{2} \mu \mid\right.$ $\left(l_{1} \rightarrow r_{1}, p, l_{2} \rightarrow r_{2}\right)_{\mu}$ is an overlap of $\left.\mathcal{R}\right\}$ yields a correct but strictly weaker confluence criterion as termination of $\mathrm{CPS}^{\prime}(\mathcal{R}) / \mathcal{R}$ implies termination of $\mathrm{CPS}(\mathcal{R}) / \mathcal{R}$ but not vice versa; $\operatorname{CPS}^{\prime}\left(\mathcal{R}^{\prime}\right) / \mathcal{R}^{\prime}$ in Example 17 is not terminating.

The next example explains why one cannot replace $\operatorname{CPS}(\mathcal{R})$ by one of its subsets $\mathrm{CPS}_{o}(\mathcal{R})=\left\{l_{2} \mu \rightarrow r_{2} \mu \mid\left(l_{1} \rightarrow r_{1}, p, l_{2} \rightarrow r_{2}\right)_{\mu}\right.$ is an overlap of $\left.\mathcal{R}\right\}$ and $\mathrm{CPS}_{i}(\mathcal{R})=\left\{l_{2} \mu \rightarrow l_{2} \mu\left[r_{1} \mu\right]_{p} \mid\left(l_{1} \rightarrow r_{1}, p, l_{2} \rightarrow r_{2}\right)_{\mu}\right.$ is an overlap of $\left.\mathcal{R}\right\}$.

Example 18. Consider the TRSs $\mathcal{R}_{1}=\{\mathrm{f}(\mathrm{a}) \rightarrow \mathrm{c}, \mathrm{f}(\mathrm{b}) \rightarrow \mathrm{d}, \mathrm{a} \rightarrow \mathrm{b}, \mathrm{b} \rightarrow \mathrm{a}\}$ and $\mathcal{R}_{2}=\{\mathrm{a} \rightarrow \mathrm{c}, \mathrm{b} \rightarrow \mathrm{d}, \mathrm{f}(\mathrm{a}) \rightarrow \mathrm{f}(\mathrm{b}), \mathrm{f}(\mathrm{b}) \rightarrow \mathrm{f}(\mathrm{a})\}$. Both TRSs are locally confluent but not confluent. We have $\mathrm{CPS}_{o}\left(\mathcal{R}_{1}\right)=\{\mathrm{f}(\mathrm{a}) \rightarrow \mathrm{c}, \mathrm{f}(\mathrm{b}) \rightarrow \mathrm{d}\}$ and $\mathrm{CPS}_{i}\left(\mathcal{R}_{2}\right)=\{\mathrm{f}(\mathrm{a}) \rightarrow \mathrm{f}(\mathrm{c}), \mathrm{f}(\mathrm{b}) \rightarrow \mathrm{f}(\mathrm{d})\}$. It is easy to see that $\mathrm{CPS}_{o}\left(\mathcal{R}_{1}\right) / \mathcal{R}_{1}$ and $\mathrm{CPS}_{i}\left(\mathcal{R}_{2}\right) / \mathcal{R}_{2}$ are terminating.

An easy extension of our main result is obtained by excluding critical pair steps from $\operatorname{CPS}(\mathcal{R})$ that give rise to trivial critical pair steps. The proof is based on the observation that Lemma 12 still holds for this modification of $\operatorname{CPS}(\mathcal{R})$.

\section{Automation}

Concerning the automation of Theorem 16, for checking relative termination we use the following criteria of Geser [8]:

Theorem 19. For TRSs $\mathcal{R}$ and $\mathcal{S}, \mathcal{R} / \mathcal{S}$ is terminating if

1. $\mathcal{R}=\varnothing$,

2. $\mathcal{R} \cup \mathcal{S}$ is terminating, or

3. there exist a well-founded order $>$ and a quasi-order $\geqslant$ such that $>$ and $\geqslant$ are closed under contexts and substitutions, $\geqslant \cdot>\cdot \geqslant \subseteq>, \mathcal{R} \cup \mathcal{S} \subseteq \geqslant$, and $(\mathcal{R} \backslash>) /(\mathcal{S} \backslash>)$ is terminating.

Based on this result, termination of $\operatorname{CPS}(\mathcal{R}) / \mathcal{R}$ is shown by repeatedly using the last condition to simplify $\mathrm{CPS}(\mathcal{R})$ and $\mathcal{R}$. As soon as the first condition applies, termination is concluded. If the first condition does not apply and the third condition does not make progress, we try to establish termination of $\mathrm{CPS}(\mathcal{R}) \cup \mathcal{R}$ using the termination tool $\mathrm{T}_{\mathrm{T}} \mathrm{T}_{2}[15]$. For checking the third condition we use matrix interpretations [7].

In the remainder of this section we show how to implement Theorem 3. We start by observing that the condition of Theorem 3 is undecidable even for locally confluent TRSs.

Lemma 20. The following decision problem is undecidable: 
instance: a finite locally confluent linear TRS $\mathcal{R}$,

question: are all critical pairs locally decreasing with respect to the rule-labeling heuristic?

Proof. We provide a reduction from the problem whether two (arbitrary) ground terms in a linear non-overlapping TRS are joinable. The latter is undecidable as an easy consequence of the encoding of Turing machines as linear nonoverlapping TRSs, see e.g. [18]. So let $\mathcal{S}$ be a (finite) linear non-overlapping TRS and let $s$ and $t$ be arbitrary ground terms. We extend $\mathcal{S}$ with fresh constants $\mathrm{a}, \mathrm{b}$ and the rewrite rules $\{\mathrm{a} \rightarrow s, \mathrm{~b} \rightarrow t, \mathrm{a} \rightarrow \mathrm{b}, \mathrm{b} \rightarrow \mathrm{a}\}$ to obtain the TRS $\mathcal{R}$. If $s$ and $t$ are joinable (in $\mathcal{S}$ ) then all its all critical pairs are locally decreasing by ordering all rules in $\mathcal{S}$ below the above four rules If $s$ and $t$ are not joinable, then no order on the rules will make the critical pairs locally decreasing with respect to the rule-labeling heuristic. So confluence of $\mathcal{R}$ can be established by the rule-labeling heuristic if and only if the terms $s$ and $t$ are joinable in $\mathcal{S}$.

By putting a bound on the number of steps to check joinability we obtain a decidable condition for (extended) local decreasingness:

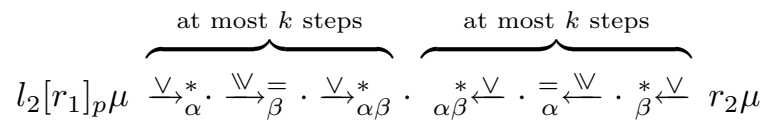

for each overlap $\left(l_{1} \rightarrow r_{1}, p, l_{2} \rightarrow r_{2}\right)_{\mu}$ of $\mathcal{R}$ with $\alpha=l_{1} \rightarrow r_{1}$ and $\beta=l_{2} \rightarrow r_{2}$. Below we reduce this to precedence constraints of the form

$$
\phi::=\top|\perp| \phi \vee \phi|\phi \wedge \phi| \alpha>\alpha \mid \alpha \geqslant \alpha
$$

where $\alpha$ stands for variables corresponding to the rules in $\mathcal{R}$. From the encodings of termination methods for term rewriting, we know that the satisfiability of such precedence constraints is easily determined by SAT or SMT solvers (cf. [4,23]).

Definition 21. For terms $s$, $t$ and $k \geqslant 0$, a pair $\left(\left(\gamma_{1}, \ldots, \gamma_{m}\right),\left(\delta_{1}, \ldots, \delta_{n}\right)\right)$ is called a $k$-join instance of $(s, t)$ if $m, n \leqslant k, \gamma_{1}, \ldots, \gamma_{m}, \delta_{1}, \ldots, \delta_{n} \in \mathcal{R}$, and

$$
s \rightarrow \gamma_{1} \cdots \cdots \rightarrow_{\gamma_{m}} \cdot \delta_{n} \leftarrow \cdot \cdots \delta_{\delta_{1}} \leftarrow t
$$

The embedding order $\sqsupseteq$ on sequences is defined as $\left(a_{1}, \ldots, a_{n}\right) \sqsupseteq\left(a_{i_{1}}, \ldots, a_{i_{m}}\right)$ whenever $1 \leqslant i_{1}<\cdots<i_{m} \leqslant n$. The set of all minimal (with respect to $\sqsupseteq \times \sqsupseteq$ ) $k$-join instances of $(s, t)$ is denoted by $J_{k}(s, t)$. We define $\Phi_{\beta}^{\alpha}\left(\left(\gamma_{1}, \ldots, \gamma_{n}\right)\right)$ as

$$
\bigvee_{i \leqslant n}\left(\bigwedge_{j<i} \alpha>\gamma_{j} \wedge \Psi_{i, n}\right)
$$

with $\Psi_{i, n}$ denoting $\top$ if $i=n$ and

$$
\beta \geqslant \gamma_{i} \wedge \bigwedge_{i<j \leqslant n}\left(\alpha>\gamma_{j} \vee \beta>\gamma_{j}\right)
$$


if $i<n$. Furthermore, $\mathrm{RL}_{k}(\mathcal{R})$ denotes the conjunction of

$$
\bigvee\left\{\Phi_{l_{2} \rightarrow r_{2}}^{l_{1} \rightarrow r_{1}}(\gamma) \wedge \Phi_{l_{1} \rightarrow r_{1}}^{l_{2} \rightarrow r_{2}}(\boldsymbol{\delta}) \mid(\boldsymbol{\gamma}, \boldsymbol{\delta}) \in J_{k}\left(l_{2}\left[r_{1}\right]_{p} \mu, r_{2} \mu\right)\right\}
$$

for all overlaps $\left(l_{1} \rightarrow r_{1}, p, l_{2} \rightarrow r_{2}\right)_{\mu}$ of $\mathcal{R}$.

The only non-trivial part of the encoding is the minimality condition in $J_{k}(s, t)$. The next lemma explains why non-minimal pairs can be excluded from the set and Example 23 shows the benefit of doing so.

Lemma 22. If $\Phi_{\beta}^{\alpha}(\boldsymbol{\delta})$ is satisfiable and $\boldsymbol{\delta} \sqsupseteq \boldsymbol{\gamma}$ then $\Phi_{\beta}^{\alpha}(\boldsymbol{\gamma})$ is satisfiable.

Proof. Straightforward.

We illustrate the encoding on a concrete example.

Example 23. Consider the TRS $\mathcal{R}$ of Example 4. We show how $\mathrm{RL}_{4}(\mathcal{R})$ is computed. There is a single overlap $(1,11,5)_{\epsilon}$ resulting in the critical pair $s \leftarrow \rtimes \rightarrow t$ with $s=\operatorname{inc}(\operatorname{tl}(0: \operatorname{inc}($ nats $)))$ and $t=\operatorname{tl}(\operatorname{inc}($ nats $))$. Its 4 -join instances are

$$
\begin{array}{llll}
((3),(1,4,3)) & ((3,1),(1,4,3,1)) & ((3,1),(1,4,1,3)) & ((3,1),(1,1,4,3)) \\
& ((1,3),(1,4,3,1)) & ((1,3),(1,4,1,3)) & ((1,3),(1,1,4,3))
\end{array}
$$

Only the first one belongs to $J_{4}(s, t)$ and hence $\mathrm{RL}_{4}(\mathcal{R})=\Phi_{5}^{1}((3)) \wedge \Phi_{1}^{5}((1,4,3))$ with $\Phi_{5}^{1}((3))=5 \geqslant 3 \vee 1>3$ and

$$
\begin{gathered}
\Phi_{1}^{5}((1,4,3))=(1 \geqslant 1 \wedge(1>4 \vee 5>4) \wedge(1>3 \vee 5>3)) \\
\vee(5>1 \wedge 1 \geqslant 4 \wedge(1>3 \vee 5>3)) \vee(5>1 \wedge 5>4 \wedge 1 \geqslant 3) \\
\quad \vee(5>1 \wedge 5>4 \wedge 5>3)
\end{gathered}
$$

This formula is satisfied by taking (e.g.) the order $5>3,4$. Hence, the confluence of $\mathcal{R}$ is concluded by local decreasingness with respect to the rule labeling heuristic using at most 3 steps to close critical pairs.

Theorem 24. A linear TRS $\mathcal{R}$ is confluent if $\mathrm{RL}_{k}(\mathcal{R})$ is satisfiable for some $k \geqslant 0$.

\section{Experimental Results}

We tested our methods on a collection of 425 TRSs, consisting of the 103 TRSs in the ACP distribution, ${ }^{5}$ the TRSs of Examples 5, 17, and 18, and those TRSs in version 5.0 of the Termination Problems Data Base $^{6}$ that are either nonterminating or not known to be terminating. (Systems that have extra variables in right-hand sides of rewrite rules are excluded.) The results are summarized in Table 1. The following techniques are used to produce the columns: (a) Knuth

\footnotetext{
${ }^{5}$ http://www nue.riec.tohoku.ac.jp/tools/acp/

6 http://termination-portal.org/wiki/TPDB
} 


\begin{tabular}{lrrrrrrr}
\hline & (a) & $(\mathrm{b})$ & $(\mathrm{c})$ & $(\mathrm{d})$ & $(\mathrm{e})$ & $(\mathrm{f})$ & $(\mathrm{g})$ \\
& 20 & 81 & 67 & 49 & 100 & 107 & 135 \\
YES & 0 & 0 & 0 & 3 & 4 & 3 & 17 \\
timeout $(60 \mathrm{~s})$ & & & & & &
\end{tabular}

Table 1. Summary of experimental results

and Bendix' criterion [14]: termination and joinability of all critical pairs, (b) orthogonality, (c) Theorem 24 with $k=4$, (d) Theorem 6, (e) Theorem 16, (f) the extension of Theorem 16 mentioned at the end of Section 3 in which critical pair steps that generate trivial critical pairs are excluded from $\operatorname{CPS}(\mathcal{R})$, and (g) ACP [2]. To obtain the data in columns (a)-(f) we slightly extended the open source termination tool $\mathrm{T}_{\mathrm{T}} \mathrm{T}_{2}$. For the data in column (c) the SAT solver MiniSat [6] is used. Since local confluence is undecidable (for non-terminating TRSs), in (c)-(f) it is approximated by $\leftarrow \rtimes \rightarrow \subseteq \bigcup\left\{\rightarrow^{i} \cdot{ }^{j} \leftarrow \mid i, j \leqslant 4\right\}$.

ACP proves that 198 of the 424 TRSs are not confluent. Of the remaining 226 TRSs, local confluence can be shown using at most 4 rewrite steps from both terms in every critical pair for 187 TRSs. Moreover, of these 187 TRSs, 148 are left-linear and 76 are linear. As a final remark, the combination of (c) and (f) proves that 129 TRSs are confluent, (a)+(c)+(f) shows confluence for 134 TRSs, and $(\mathrm{c})+(\mathrm{f})+(\mathrm{g})$ shows confluence for 145 TRSs. These numbers clearly show that both our results have a role to play.

\section{Conclusion}

In this paper we presented two results based on the decreasing diagrams technique for proving confluence of TRSs. For linear TRSs we showed how the rulelabeling heuristic can be implemented by means of an encoding as a satisfiability problem and we employed the self-labeling heuristic to obtain the result that an arbitrary left-linear locally confluent TRS is confluent if its critical pair steps are relatively terminating with respect to its rewrite rules. We expect that both results will increase the power of ACP [2].

As future work we plan to investigate whether the latter result can be strengthened by decreasing the set CPS $(\mathcal{R})$ of critical pair steps that need to be relatively terminating with respect to $\mathcal{R}$. We anticipate that some of the many critical pair criteria for confluence that have been proposed in the literature (e.g. $[11,21])$ can be used for this purpose. The idea here is to exclude the critical pair steps that give rise to critical pairs whose joinability can be shown by the conditions of the considered criterion. Another direction for future work is to determine whether the conversion version of decreasing diagrams [22] can increase the power of automatic confluence tools. Last but not least, in order to certify the output of such tools, we plan to formalize the confluence results presented in this paper in the Isabelle proof assistant. 
Acknowledgements. We thank Mizuhito Ogawa, Vincent van Oostrom, and Harald Zankl for commenting on an earlier version of this paper. The detailed comments of the anonymous referees helped to improve the paper.

\section{References}

1. Aoto, T., Toyama, Y.: Persistency of confluence. Journal of Universal Computer Science 3(11), 1134-1147 (1997)

2. Aoto, T., Yoshida, J., Toyama, Y.: Proving confluence of term rewriting systems automatically. In: Proc. 20th RTA. LNCS, vol. 5595, pp. 93-102 (2009)

3. Bezem, M., Klop, J., van Oostrom, V.: Diagram techniques for confluence. I\&C 141(2), 172-204 (1998)

4. Codish, M., Lagoon, V., Stuckey, P.: Solving partial order constraints for LPO termination. In: Proc. 17th RTA. LNCS, vol. 4098, pp. 4-18 (2006)

5. Dershowitz, N.: Open. Closed. Open. In: Proc. 16th RTA. LNCS, vol. 3467, pp. 276-393 (2005)

6. Eén, N., Sörensson, N.: An extensible SAT-solver. In: Proc. 6th SAT. LNCS, vol. 2919, pp. 502-518 (2004)

7. Endrullis, J., Waldmann, J., Zantema, H.: Matrix interpretations for proving termination of term rewriting. JAR 40(2-3), 195-220 (2008)

8. Geser, A.: Relative Termination. PhD thesis, Universität Passau (1990). Available as technical report 91-03.

9. Gramlich, B.: Termination and Confluence Properties of Structured Rewrite Systems. PhD thesis, Universität Kaiserslautern (1996)

10. Gramlich, B., Lucas, S.: Generalizing Newman's lemma for left-linear rewrite systems. In: Proc. 17th RTA. LNCS, vol. 4098, pp. 66-80 (2006)

11. Huet, G.: Confluent reductions: Abstract properties and applications to term rewriting systems. J. ACM 27(4), 797-821 (1980)

12. Jouannaud, J.P., van Oostrom, V.: Diagrammatic confluence and completion. In: Proc. 36th ICALP. LNCS, vol. 5556, pp. 212-222 (2009)

13. Klop, J., van Oostrom, V., de Vrijer, R.: A geometric proof of confluence by decreasing diagrams. Journal of Logic and Computation 10(3), 437-460 (2000)

14. Knuth, D., Bendix, P.: Simple word problems in universal algebras. In: Computational Problems in Abstract Algebra. Pergamon Press 263-297 (1970)

15. Korp, M., Sternagel, C., Zankl, H., Middeldorp, A.: Tyrolean termination tool 2. In: Proc. 20th RTA. LNCS, vol. 5595, pp. 295-304 (2009)

16. Ohlebusch, E.: Modular Properties of Composable Term Rewriting Systems. PhD thesis, Universität Bielefeld (1994)

17. Rosen, B.: Tree-manipulating systems and Church-Rosser theorems. J. ACM 20(1), 160-187 (1973)

18. Terese: Term Rewriting Systems. vol. 55 of Cambridge Tracts in Theoretical Computer Science. Cambridge University Press (2003)

19. Toyama, Y.: Commutativity of term rewriting systems. In: Programming of Future Generation Computers II. North-Holland 393-407 (1988)

20. van Oostrom, V.: Confluence by decreasing diagrams. TCS 126(2), 259-280 (1994)

21. van Oostrom, V.: Developing developments. TCS 175(1), 159-181 (1997)

22. van Oostrom, V.: Confluence by decreasing diagrams converted. In: Proc. 19th RTA. LNCS, vol. 5117, pp. 306-320 (2008)

23. Zankl, H., Hirokawa, N., Middeldorp, A.: KBO orientability. JAR 43(2), 173-201 (2009) 